\title{
44543 - CONTINUOUS CELL SAVER REDUCES COGNITIVE DECLINE AFTER CABG SURGERY
}

Disclosure: Grants/research support - Heart \& Stroke Foundation of Ontario

\author{
George Djaiani, Toronto General Hospital, University Health Network, Toronto, ON, \\ Canada; \\ Ludwik Fedorko, Toronto General Hospital, University Health Network; \\ Michael Borger, Toronto General Hospital, University Health Network; \\ $R$ Green, Toronto Rehabilitation Centre, University of Toronto; \\ J Carroll, Toronto General Hospital, University Health Network; \\ BJ Anderson, Toronto General Hospital, University Health Network; \\ M Marcon; \\ J Karski, Toronto General Hospital, University Health Network;
}

INTRODUCTION: Blood collected in cardiotomy suction contains high levels of cellular debris and lipid microparticulates, which have been shown to cause embolization of the brain blood vessels. If shed blood was processed with a cell-saver device, it considerably reduced cerebral lipid embolization. The objective of this study was to determine if replacement of cardiotomy suction with a continuous flow cell-saver device would reduce cognitive decline in elderly patients after elective CABG surgery.

METHODS: After REB approval and informed consent, 226 patients over 60 years were randomly allocated to either cell saver or control (i.e. cardiotomy suction) groups.

Neuropsychological testing was conducted 1 week before (baseline) and 6-weeks after surgery by a psychometrist blinded to the treatment arm assignment. The proposed battery of tests complied with the international consensus on assessing neuropsychological outcome. A Z-score was calculated for each of the 10 main test variables in each patient. For cognitive dysfunction to be considered significant it had to occur in two or more tests. Anesthesia and surgical management was standardized. A comprehensive TEE examination and epiaortic scanning were performed in all patients. A subset of patients in each group underwent transcranial Doppler (TCD) assessments to detect cerebral embolic load.

RESULTS: There was no difference with baseline demographic data, preoperative variables, and surgical characteristics between the two groups. Cognitive outcome could not be determined at 6 weeks in 13 and 15 patients in the cell-saver and control groups respectively. Baseline neuropsychological test scores were similar between the two groups. Cognitive dysfunction was present in $6 \%$ (95\% CI $1.3 \%$ to $10.7 \%$ ) of patients in the cell saver group and $15 \%$ (95\% CI $8 \%$ to $22 \%)$ of patients in the control group. $(\mathrm{p}=0.038)$. The rates of improvement above the $1 \mathrm{SD}$ were similar between the two groups; $19 \%$ (95\% CI $11.4 \%$ to $26.6 \%)$ in the cell saver group versus $17 \%$ (95\% CI $9.8 \%$ to $24.2 \%$ ) in the control group ( $\mathrm{p}=0.712$ )(Figure) Using the raw Zscores as a continuous cognitive outcome, patients in the cell saver group performed considerably better on three of the ten variables assessed. The severity and distribution of atheroma were similar between the two groups. There was no significant difference with respect to the TCD detected embolic events during CPB between the two groups. 
DISCUSSION: Replacement of cardiotomy suction with the continuous flow cell-saver is a novel approach to improve neuro-protection and enhance recovery of cognitive function in patients undergoing cardiac surgery. The present study demonstrates that 'cleansing' of the unprocessed shed blood reduces cognitive decline in elderly patients after CABG surgery.

Figure. The rates of improvement (positive bars) and deterioration (negative bars) in neuropsychological test scores after coronary artery bypass graft surgery at 6-weeks follow-up. Values shown are expressed as a percentage of patients outside one standard deviation on Z-score for each test. ${ }^{*} \mathrm{p}=0.005, * * \mathrm{p}=0.04, * * * \mathrm{p}=0.03$.

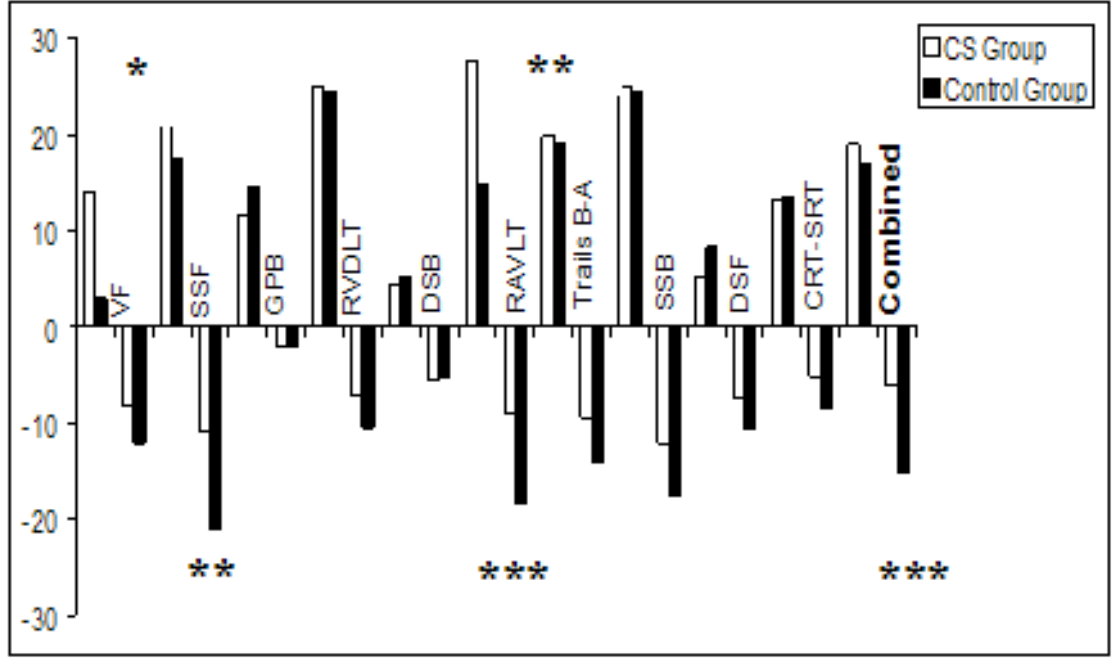

